\title{
TTR
}

Traduction, terminologie, re?daction

\section{Jeanne Dancette. Parcours de traduction. Étude expérimentale du processus de compréhension. Lille, Presses Universitaires de Lille, coll. " Étude de la traduction », 1995, 256 p.}

\section{Danielle Boissinot}

Volume 9, numéro 1, 1er semestre 1996

Le festin de Babel

Babel's Feast

URI : https://id.erudit.org/iderudit/037250ar

DOI : https://doi.org/10.7202/037250ar

Aller au sommaire du numéro

Éditeur(s)

Association canadienne de traductologie

ISSN

0835-8443 (imprimé)

1708-2188 (numérique)

Découvrir la revue

Citer ce compte rendu

Boissinot, D. (1996). Compte rendu de [Jeanne Dancette. Parcours de traduction. Étude expérimentale du processus de compréhension. Lille, Presses

Universitaires de Lille, coll. "Étude de la traduction ", 1995, 256 p.] TTR, 9(1),

304-309. https://doi.org/10.7202/037250ar d'utilisation que vous pouvez consulter en ligne.

https://apropos.erudit.org/fr/usagers/politique-dutilisation/ 
Jeanne Dancette. Parcours de traduction. Étude expérimentale du processus de compréhension. Lille, Presses Universitaires de Lille, coll. « Étude de la traduction ", 1995, $256 \mathrm{p}$.

Issu de la thèse de doctorat que Jeanne Dancette a soutenue à l'Université de Montréal, Parcours de traduction est une contribution stimulante à l'étude du processus de construction du sens au cours de l'activité traduisante. L'auteure a voulu explorer la notion de compréhension ainsi que les liens entre la saisie du sens d'un texte et l'adéquation sémantique de la traduction qui en est faite, ou, en d'autres termes, de vérifier l'exactitude de l'axiome selon lequel « pour traduire, il faut comprendre». Par ailleurs, soucieuse d'ancrer l'analyse de la compréhension dans la réalité et d'en assurer le plus possible l'objectivité et le caractère scientifique, J. Dancette a opté pour une approche expérimentale, recueilli des données d'observation et recouru pour les analyser à des modèles sémantiques et cognitifs. À long terme, elle espère que les études comme la sienne conduiront à l'élaboration de nouveaux outils pour l'enseignement de la traduction.

La première partie de l'ouvrage est réservée aux considérations d'ordre théorique. Après un survol de la place qu'occupe le problème crucial de la compréhension dans les diverses disciplines qui s'intéressent au langage, une cinquantaine de pages sont consacrées à de nombreux états de questions se rapportant 1) aux théories du sens : approche linguistique du sens dans deux modèles de la traduction, modèles conceptuels du sens ; 2) à la position des théoriciens et des didacticiens de la traduction à l'égard de la compréhension : selon le type de traduction (textes techniques, littéraires...) qu'ils étudient, selon qu'ils adoptent une approche « par la négative" (sanction des erreurs de sens) ou une attitude " formative " (recherchedes compétencesnécessairesau traducteur), selon qu'ils considèrent la compréhension comme instantanée ou comme se construisant de façon graduelle et selon leur position quant aux rapports entre le sens et la forme ; et enfin, 3) aux 
approches empiriques de la compréhension : études d'observation de lecteurs ou de traducteurs (questions de compréhension, résumés de lecture, observation de l'introspection ou du raisonnement à voix haute de sujets en train de traduire) et tentatives de simulation de l'activité analytique de l'être humain pour la faire effectuer par la machine (traduction assistée par ordinateur, traduction automatique).

Certains points de vue ne sont présentés que de manière très succincte, ce qui fait paraître l'ensemble légèrement décousu en dépit de l'unité de thème. J. Dancette intégrant dans sa démarche les contributions de plusieurs disciplines, aucun de ces états ne peut toutefois être considéré comme inutile, bien que le développement réservé à la traduction par ordinateur paraisse quelque peu đisproportionné. Après tout, la résolution des problèmes rencontrés en analyse automatique du sens nous renseigne bien plus sur ce que l'être humain pense faire lorsqu'il comprend que sur ce qu'il fait effectivement. Et même une traduction « parfaite » réalisée par la machine ne permettrait pas de conclure que nous procédons de la même manière pour artiver au même résultat. J. Dancette formule d'ailleurs une réserve à cet effet.

La description de l'expérience à laquelle s'est livrée J. Dancette ainsi que la présentation et le commentaire des résultats obtenus constituent le deuxième volet de Parcours de traduction. Les éléments de description du sens qui ont été empruntés aux approches linguistique et cognitive sont d'abord examinés en détail. L'auteure considère que l'arbre syntaxique de la grammaire générative peut être utilisé avec profit pour représenter l'organisation syntagmatique de la phrase, ou, en cas d'ambiguité, les diverses possibilités, et que, par ailleurs, la construction d'un graphe sémantique, à la manière de Mel'čuk, fournit une représentation des relations prédicatives dont il est possible de tirer grand parti. Arbres syntaxiques et, surtout, graphes sémantiques ont été employés au cours de l'évaluation des traductions et des réponses aux questions de compréhension pour comparer le sens des énoncés traduits ou paraphrasés et le sens posé par l'auteure comme étant celui de ces énoncés. Aux modèles cognitifs de Kintsch et Van Dijk et de R. de Beaugrande, l'auteure emprunte la notion de hiérarchisation des 
propositions; elle considère qu'une faute commise dans une proposition de niveau supérieur est plus grave qu'une faute commise dans une proposition de niveau inférieur et pondère les résultats obtenus aux questions de compréhension en fonction de cette hiérarchie. Le poids attribué à chacune des réponses dans le score total est censé refléter l'importance relative des propositions pour la compréhension globale du texte.

L'expérience réalisée par J. Dancette est fondée, d'une part, sur l'hypothèse selon laquelle, bien qu'on ne puisse pas observer directement les processus de compréhension, il est tout de même possible d'en détecter des indices et de les analyser, et, d'autre part, sur une définition de la compréhension selon laquelle celle-ci est " ... le résultat de la confrontation et de la synthèse du sens littéral et du sens contextuel. [Le premier est] porté par les éléments linguistiques [et le second] par l'acte de communication et élaboré à partir des interprétations faites sur le sens de l'énoncé, et des inférences et déductions faites sur les connaissances extralinguistiques et encyclopédiques " (p. 82). En conséquence, des étudiants francophones à la maîtrise en traduction ont été soumis à quatre tests : questions de compréhension sur le texte à traduire (paraphrases, explicitations des rapports entre les propositions...) afin de vérifier que les énoncés en contexte étaient compris, traduction d'un court texte ayant trait aux élections américaines, test de compétence linguistique élaboré par l'auteure afin d'évaluer la connaissance globale de l'anglais et, finalement, test de connaissances relatives au thème général de la traduction.

Ces tests fournissent une abondance de données commentées en détail par l'auteure. L'une des questions de compréhension présente un intérêt particulier du fait qu'elle illustre la difficulté de déterminer si la compréhension repose sur un élément linguistique ou sur des déductions faites à partir de connaissances extralinguistiques. Le texte de la traduction se terminait par la phrase suivante : "... Sen. Albert Gore, who did well on Super Tuesday but proved to be no Crocodile Dundee in the jungles of New York" (p. 113), et les sujets de l'expérience ont été priés d'expliquer ce que signifie "to be no Crocodile Dundee... ". Six des treize 
personnes qui ont donné une réponse satisfaisante n'avaient pas vu le film, tandis que quatre des six personnes qui avaient vu le film ont donné une réponse insatisfaisante. J. Dancette semble penser (p. 124 et 193) que ceux qui ont vu le film raisonnent à partir de ce qu'ils savent du personnage et que l'inférence à partir de «but » n'est utilisée que par ceux qui ne peuvent faire autrement. Rien n'est moins sûr. En fait, l'inférence était la seule manière de procéder pour ne pas risquer de substituer sa propre vision de Crocodile Dundee, à supposer qu'elle soit négative, à celle du texte. (Ce qui a dî se produire pour les personnes qui ont vu le film, mais donné une réponse fautive.) Un meilleur exemple de la possibilité, soulignée par J. Dancette (p. 192), de suppléer à une lacune dans un ordre de connaissances en recourant à un autre niveau d'analyse est donné par l'abréviation "Sen. ». Source importante d'erreurs dans la traduction, la méconnaissance de l'abréviation a conduit les deux cinquièmes des sujets de l'expérience à prendre le point pour un point de fin de phrase ou "Sen » pour un prénom ou un surnom. La faute a été considérée comme étant d'ordre linguistique (connaissance du code typographique), mais le problème peut parfaitement être résolu par déduction à partir de connaissances sur le rôle des sénateurs dans la vie politique américaine (c'est de cette manière que j'ai procédé).

Compte tenu de la petitesse de l'échantillon, les analyses statistiques des résultats sont, avec raison, présentées sous toutes réserves. De ces analyses, se dégage une forte corrélation - qui ne peut pas être le fruit du hasard - entre le rang des sujets au test de compréhension et leur rang en traduction, ainsi qu'entre leurs compétences linguistiques et leur rang en traduction. Ces résultats ne sont pas dépourvus d'intérêt - voilà maintenant l'axiome appuyé par la recherche scientifique -, mais ils étaient assez prévisibles. La corrélation entre le rang obtenu au test de compréhension et le rang en connaissances thématiques est significative tandis que la corrélation entre le rang obtenu au test de compréhension et le rang en compétences linguistiques n'est pas statistiquement significatif. Les résultats les plus intéressants proviennent de l'analyse multivariée des corrélations : en divisant l'échantillon en deux sousgroupes à partir d'une des trois variables (disons d'un côté les 
meilleurs en compétence linguistique et, de l'autre, les moins bons), il est possible d'observer le comportement des autres variables (ici, la compréhension et les connaissances thématiques). Les résultats de cette analyse permettent de formuler l'hypothèse selon laquelle il existe un seuil minimal de connaissances linguistiques en deçà duquel les connaissances thématiques ne peuvent être mises à profit ni pour comprendre ni pour traduire ; même la relation entre la compréhension et la traduction semble dépendre de ce seuil minimal. De la même manière, il se peut qu'il existe un seuil de connaissances thématiques au-delà duquel le lien entre compétences linguistiques et compréhension est renforcé.

Une analyse qualitative des fautes de sens commises par les sujets de l'expérience fait suite à l'analyse quantitative. Les types d'erreurs sont regroupés en catégories; quatorze types de fautes relèvent de la compétence linguistique et dix-sept de capacités cognitives (inférences, déductions). La proportion d'erreurs qui relèvent des capacités cognitives est suffisamment forte pour donner à penser avec l'auteure que « les habiletés de raisonnement sont à développer autant, si ce n'est plus, que les habiletés purement linguistiques $»$ (p. 192).

L'ouvrage se termine sur un schéma qui ne découle pas directement de l'expérience dont il vient d'être question, mais plutôt des recherches empiriques sur les processus de traduction, y compris d'un enregistrement de raisonnements à voix haute réalisé par $\mathrm{J}$. Dancette. Par ce schéma en forme de double hélice l'auteure veut illustrer le processus de compréhension chez le traducteur et signifier en particulier que compréhension et recherche d'équivalences ne sont pas des phénomènes distincts et successifs, que la recherche d'équivalences est contraignante, qu'elle modifie au fur et à mesure qu'il s'y livre la compréhension du traducteur, ce qui le distingue du lecteur " ordinaire » et lui permet d'en arriver à une nouvelle équivalence et à une compréhension généralementmeilleure, et ainsi de suite jusqu'à ce qu'il juge le résultat du processus satisfaisant.

Parcours de traduction est une entreprise qui peut sans aucun doute être qualifiée d'audacieuse et qui a le mérite de sortir 
des sentiers battus et d'ouvrir des perspectives nouvelles. Il est certain que Jeanne Dancette a " vu grand "; l'ampleur du problème auquel elle s'est attaquée ainsi que le nombre de ramifications qu'elle a dû explorer et d'outils qu'il lui a fallu forger impressionnent. Elle a peut-être été amenée à combattre sur trop de fronts à la fois, mais on peut difficilement le lui reprocher : la recherche traductologique a bien trop besoin de gens aventureux comme elle.

Danielle Boissinot Université Laval 